\title{
EVALUACIÓN DE ALTERNATIVAS DE COSTO PARA EL SUMINISTRO DE PROPANTES A UN YACIMIENTO NO CONVENCIONAL EN COLOMBIA
}

Laura Daniela Garcés Carreñoํㅜ Juan Benjamín Duarte Duarte ${ }^{2 *}$, Edwin Alberto Garavito Hernández ${ }^{3}$, Darwin Clemente Mateus Tarazona ${ }^{4}$, Edgar Ricardo Pérez Carrillo ${ }^{5}$

* A quien debe dirigirse la correspondencia

\section{RESUMEN}

El suministro de propantes a yacimientos no convencionales es uno de los mayores desafíos que enfrentan las empresas petroleras para completar los pozos a tiempo, buscando minimizar los costos. Teniendo en cuenta esto, en el presente trabajo se busca plantear alternativas para abastecer el material apuntalante al pozo, y evaluar a través de simulación Monte Carlo cuál de ellas es la mejor basados en el criterio de menor costo. Los resultados muestran que bajo las condiciones y supuestos dados, la mejor alternativa es la de importación directa por parte de la empresa operadora, teniendo en cuenta que su costo es más bajo comparado con las opciones de comprarlo a una compañía de servicio técnico o a un intermediario importador.

Palabras clave: yacimientos no convencionales, propantes, simulación de Monte Carlo.

\section{EVALUATING COST ALTERNATIVES FOR SUPPLYING PROPPANTS TO AN UNCONVENTIONAL RESERVOIR IN COLOMBIA}

\begin{abstract}
The supply of proppants to unconventional reservoirs is one of the biggest challenges that oil companies are facing in order to complete wells on time while minimizing costs. With this in mind, this article seeks to propose alternatives for supplying proppants to wells and to develop an evaluation using Monte Carlo simulation, which is one of the best tools based in lowest cost criteria. Results of this research show how under the given conditions and estimations, the best alternative the direct import is done by the oil company, considering that the cost is lower compared with other options such as buying the oil from a technical service company or from a third-party import company.
\end{abstract}

Keywords: unconventional reservoirs, proppants, Monte Carlo Simulation.

1. Ingeniera Industrial. Candidata a M.Sc. en Ingeniería Industrial de la Universidad Industrial de Santander. lauradgarces@gmail.com. Bucaramanga-Colombia

2. Doctor en Finanzas de Empresa de la Universidad Complutense de Madrid, Profesor titular Escuela de Estudios Industriales y Empresariales, Universidad Industrial de Santander. jbduarted@hotmail.com. BucaramangaColombia

3. Ingeniero Industrial. Especialista en Gerencia de la Producción y Mejoramiento Continuo. Candidato a M.Sc. en Ingeniería Industrial. Profesor Asociado Escuela de Estudios Industriales y Empresariales, Universidad Industrial de Santander. garavito@uis.edu.co. Bucaramanga-Colombia

4. M.Sc. en Ingeniería Civil. Pontificia Universidad Católica de Rio de Janeiro. Geólogo. Instituto Colombiano del Petróleo (Ecopetrol S.A.). darwin.mateus@ecopetrol.com.co. Piedecuesta-Colombia

5. M.Sc. en Ingeniería de Hidrocarburos. Universidad Industrial de Santander. Ingeniero de petróleos. Instituto Colombiano del Petróleo (Ecopetrol S.A.).ricardo.perez@ecopetrol.com.co. Piedecuesta-Colombia 


\section{INTRODUCCIÓN}

La explotación petrolífera tradicionalmente se ha ejecutado en yacimientos convencionales, sin embargo, en los últimos años se han desarrollado técnicas de explotación para extraer gas y petróleo en yacimientos no convencionales, entre las cuales se encuentra el fracturamiento hidráulico, que consiste en generar grietas en la roca mediante la inyección a alta presión de agua mezclada con agentes de sostén y químicos, buscando que el gas y el petróleo fluyan hacia la superficie (Sovacool, 2014).

La creciente demanda de energía en el mundo y el agotamiento de los recursos convencionales, han obligado a los productores de hidrocarburos a incursionar en la exploración y explotación de recursos no convencionales, los cuales generan oportunidades potenciales de abastecimiento pero requieren un grado de dificultad mayor en su operación dada las condiciones de permeabilidad de la roca. Teniendo en cuenta lo anterior, la industria de Shale puede representar un aporte significativo para cubrir las necesidades de crudo y gas en Colombia a largo plazo; sin embargo, su operación está en desarrollo y es preciso tener en cuenta temas claves como: capital económico, regulaciones gubernamentales, cuidado del medio ambiente, seguridad y relaciones con la comunidad, así como los procesos logísticos asociados al desarrollo del shale plays. Este último ítem ha generado desafíos principalmente en lo relacionado con el suministro de agua y agentes de sostén para completar el pozo a tiempo y a unos costos relativamente aceptables de acuerdo al precio del crudo. Por lo tanto estos dos insumos, agua y propante, juegan un papel importante en el desarrollo de las operaciones de explotación de shale plays y concretamente para la industria petrolera, en pozos en donde se requiere este tipo de técnica para su explotación.

Teniendo en cuenta la situación actual de las operaciones de suministro de propantes en Colombia, caracterizada por la ausencia de fabricantes del propante tipo cerámico, y sin explotación de arenas para fracturamiento, se justifica el presente trabajo que busca evaluar tres alternativas de suministro de propantes, de tal forma que se pueda garantizar que este insumo esté disponible al menor costo posible, y así contar con precios competitivos para la comercialización del crudo a nivel mundial. La primera alternativa de suministro es a través de una compañía de servicio técnico (CST), la cual se encargaría de importar el propante, almacenarlo y bombearlo en situ; la segunda alternativa es por medio de un intermediario en Colombia, quien realizaría la importación y posiblemente el almacenamiento; y por último, la tercera alternativa se centra en evaluar la operación de importación por parte de la empresa operadora del yacimiento no convencional, la cual se encargaría de las operaciones de compra al proveedor internacional (China-USA), tramites portuarios, transporte internacional y nacional, almacenamiento, administración y bombeo in situ.

\section{PROPANTE}

De acuerdo con Caballero \& Carrillo (2012), el apuntalante, agente de sostén o propante es el material que permanece en la fractura manteniéndola abierta, estableciendo un canal conductivo para la afluencia de fluido en el pozo. Un propante ideal debería producir una máxima permeabilidad en la fractura en función de propiedades como la redondez, la esfericidad del grano, la resistencia al aplastamiento, la conductividad, entre otras. Generalmente mayores volúmenes de propante inyectados permiten más fracturas y proporcionan un flujo de retorno más rápido en el pozo productor.

Teniendo en cuenta lo enunciado por Serrano, Pineda, Corzo, \& Castillo (2013) se pueden distinguir dos categorías de material propante: naturales y elaborados. Los primeros se refieren a la arena que es uno de los agentes de sostén más utilizados en las operaciones de fracturamiento hidráulico, cuya calidad depende de la cantera y de la eficiencia del fabricante. Los segundos comprenden apuntalantes recubiertos con resina que permiten aumentar su resistencia al aplastamiento, adicionalmente existen los propantes artificiales fabricados a partir de materiales cerámicos, que proporcionan una mayor conductividad y una baja deformación en el pozo, esto gracias a que sus esferas son prácticamente perfectas.

\section{1 CADENA DE SUMINISTRO DE PROPANTES}

De acuerdo con información publicada por la consultora PacWest Consulting Partners (2014), el mercado de la arena para fracturamiento se compone principalmente de mercados locales en los países donde se explotan los recursos no convencionales, ya que cuentan con canteras para su extracción y facilidades para su transporte, proporcionando un costo relativamente bajo; en el caso de los propantes resinados está compuesto por mercados nacionales, por importaciones desde Norte América y en menor medida desde otros países productores de este tipo de material; por último, el mercado de los propantes cerámicos, que a pesar de ser los más costosos, poseen 
una alta demanda a nivel mundial dadas sus excelentes propiedades de fractura y resistencia al aplastamiento, cuenta entre los principales productores con Norte América y China.

En países como Argentina, donde recientemente se ha incursionado en operaciones de fracturamiento hidráulico, el mercado de propantes no esta tan consolidado, por lo cual parte de la arena natural y resinada, así como la totalidad de los cerámicos se importan de Estados Unidos, China y Brasil (Aguirre, 2013). Debido a los depósitos naturales descubiertos, la parte restante de arena natural es abastecida por empresas argentinas (Minera líder y Goldinka Resources S.A), que mediante diferentes técnicas han logrado tamaños de grano aptos para el desarrollo de las actividades de fractura, adicionalmente según U308CORP (2014) se han encontrado canteras de arena en Santa LuciaUruguay con propiedades que superan las normas ISO 13503-2, y que podría ser fuente de aprovisionamiento de esta materia prima a través de los puertos en Montevideo (Uruguay) y San Antonio del oeste (Argentina). Esto último aporta ventajas de logística y distribución, que se evidencia en menores costos. En cuanto al transporte de arena natural de fuentes locales para este yacimiento, Aguirre (2013) manifiesta que actualmente se realiza a través de camiones con capacidad de 30 toneladas (50 camiones por día por pozo); sin embargo, para evitar que las rutas colapsen, algunas operadoras regionales han instalado lugares de acopio, siendo un ejemplo de esto la empresa Halliburton que recientemente inauguró la primera planta de almacenamiento y distribución de apuntalante, para dar soporte a sus clientes en operaciones de yacimientos no convencionales, mejorando la productividad y disminuyendo los costos. De acuerdo con lo publicado en la Revista Petroquimica (2014) la planta dispone de un depósito con tres silos de 600 toneladas de capacidad cada uno y una banda transportadora vertical de 34 metros de altura que facilita las operaciones de carga en las tolvas transportadas por los camiones, que tienen una capacidad de descarga neumática que permite cargar sistemas "mountain mover" en pozo.

Por otro lado, en Estados Unidos existe un mercado local consolidado, lo cual facilita el aprovisionamiento de esta materia prima. Por ejemplo, Barzola (2012) expresa que el shaleplay Eagle Ford tiene dos fuentes de suministro de propantes cerámicos ultralivianos, una en Georgia y otra en China; y las arenas naturales las extrae de la Región de los Grandes Lagos (Michigan, Wisconsin y Ohio). En cuanto al transporte, los productos nacionales viajan por tren hacia el sur de Texas y de ahí se transfieren en camiones para su entrega en pozo; el apuntalante cerámico que es importado desde China, viaja en camiones desde la fábrica hasta el puerto en Shanghái en bolsas de 3000 libras y de allí, por barco, hasta el puerto de Texas, desde donde lo llevan en camiones hasta un depósito y por último se lleva en camiones al pozo.

\subsection{FORMAS DE SUPLIR LA DEMANDA DE PROPANTES EN COLOMBIA}

En el caso de la principal compañía operadora en el país (Ecopetrol S.A.), el suministro de propantes es prestado por 4 Compañías de servicio técnico de fracturamiento hidráulico, las cuales son: Weatherford, Halliburton, Schlumberger y Baker. Estas realizan las operaciones de intermediación entre el fabricante y la empresa operadora. Las CST prestan a Ecopetrol el servicio de suministro de propantes así como el alquiler de los equipos, la movilización de estos, el servicio de fracturamiento hidráulico y las operaciones de bombeo. Por lo tanto Ecopetrol incurre en unos costos asociados al alquiler de los equipos (set de fractura, equipos de bombeo, entre otros), el precio del propante por libra en dólares de acuerdo a la cantidad demandada, el costo de movilización de los equipos, transporte de los propantes dependiendo de la cantidad y la distancia desde la base de almacenamiento de la CST hasta el pozo productivo de Ecopetrol. Adicional a esto, dado que Ecopetrol actualmente se encuentra en una etapa de desarrollo en campos Shale Play y no cuenta con una estimación exacta de la cantidad de propante que requerirá para las operaciones de fracturamiento hidráulico de estos yacimientos, las CST le permiten realizar la devolución del propante que no utilice, siempre y cuando Ecopetrol cubra el costo de movilizarlo hasta la base de éste.

En cuanto a estudios que aborden alternativas de suministro de propantes en Colombia, se encontró un caso publicado por Toncel (2013) en el que evaluó cuatro posibles alternativas de suplir la demanda de propantes en Colombia, las cuales consistían en: compra de arena importada a las compañías de servicio técnico, compra de arena nacional a las compañías de servicio técnico, compra de arena importada a un intermediario y compra de arena nacional a un intermediario. Para comparar estas cuatro opciones usó un criterio de costo presente equivalente (CPE) teniendo en cuenta un consumo estimado de propante tipo arena para un horizonte de tres años y una tasa de descuento del $11,2 \%$. De acuerdo con sus resultados la opción más favorable desde el punto de vista financiero era la compra de arena nacional a las compañías de servicio Técnico, con un 
CPE de \$10.416.949; no obstante desde el punto de vista técnico no es posible llevarla a cabo, dado que en Colombia según algunos estudios realizados no se cuenta con la calidad de material adecuada (resistencia al aplastamiento) y la industria productora de arena aún está por desarrollarse. Dado lo anterior, el autor planteó como la opción más viable la compra de arena importada a un intermediario, con un CPE de 11.068.109.

\subsection{GESTIÓN DE LA CADENA DE SUMINISTRO DE PROPANTES MEDIANTE SIMULACIÓN}

El aumento de los destinos y del volumen de propante demandado, hace que la gestión de su cadena de suministro se convierta en un foco de estudio. Uno de los puntos importantes que contribuyen a un manejo adecuado de ésta, tiene que ver con la comprensión de los costos totales asociados a su operación logística. El conocimiento de estos, puede ayudar a los proveedores de servicios y materiales y a los operadores (oferentes y demandantes) a llegar a un acuerdo que los beneficie mutuamente.

Según Mawet, Fleming, \& Nichols (2012) la simulación es considerada una herramienta notable a la hora de evaluar el grado de sensibilidad que aportan variables tales como: los precios de los materiales, los medios de transporte y los sitios de perforación, sobre los costos totales de suministro. Tres de las principales tendencias que sobresalen en materia de simulación de la cadena de suministro de propantes son: el análisis estadístico, que busca reconocer y corregir errores en los datos; la programación lineal y teoría de restricciones, que tiene como fin demostrar el impacto de los factores claves en la estructura de costos asociados al suministro de propantes y obtiene mejores conclusiones sobre valores ideales; y por último la simulación mediante Monte Carlo, que permite generar múltiples escenarios que analizan el impacto en los costos de circunstancias cambiantes y proporcionan soluciones aproximadas de lo que puede suceder y la probabilidad de que suceda.

\subsection{SIMULACIÓN POR MONTECARLO}

Las operaciones logísticas para el suministro de propantes a yacimientos no convencionales, están sujetas a fuentes de riesgo e incertidumbre, asociadas principalmente a variables como la necesidad real de propante por pozo, el número de etapas por pozo, las libras de propante por etapa y la tasa de cambio. La simulación Montecarlo es útil en estos casos, ya que permite identificar y describir las variables logísticas que mayor impacto tienen sobre el costo total mediante el uso de distribuciones de probabilidad, con el fin de obtener mediante múltiples iteraciones el comportamiento estadístico de la variable de salida, que contribuya a tomar una decisión respecto a cuál es la alternativa de menor costo que se adapta a las necesidades actuales de suministro de propantes para la empresa operadora.

Un software que permite usar simulación por Montecarlo es el@Risk, el cual trabaja como un complemento de Microsoft Excel con sofisticadas funciones en la especificación y ejecución de modelos de simulación, que permite analizar cientos de escenarios al mismo tiempo, con el propósito de contribuir a la toma de decisiones (Palisade Corporation, 2013).

\section{REQUERIMIENTOS PARA EL DESARROLLO DEL RESERVORIO DE VACA MUERTA (NEUQUÉN/ ARGENTINA)}

El Instituto de Energía (2014) desarrollo un informe en el que estimó los requerimientos logísticos, recursos materiales y humanos del reservorio de Vaca Muerta, en la provincia de Neuquén (Argentina) para el periodo 2015-2030 basado en un escenario hipotético de la producción de gas y petróleo, con el fin de que dichas necesidades sean previstas y planificadas adecuadamente de forma que se logre un desarrollo efectivo de los recursos de la provincia.

De acuerdo con los planes públicos de los operadores en Argentina y las experiencias de algunos yacimientos en Estados Unidos de América los autores determinaron el número de pozos y su perfil tipo a través de un modelo de análisis espacial de la formación, para luego mediante la combinación de estos dos establecer el número de fracturas por pozo, a partir del cual estimaron las necesidades de insumos totales por año, tales como: agua, agentes de sostén, químicos, potencia de fractura, cemento, entre otros, así como la cantidad de camiones necesarios para transportar estos materiales. Según el estudio el número de fracturas por pozo en promedio será de 11 a 16 entre los años 2020 y 2030, lo que da como resultado una demanda estimada de arena para fractura por año cercana a los 3’000.000 de toneladas para el 2021 y 7’ 500.000 de toneladas para el 2030 . 


\section{METODOLOGÍA}

Para determinar la alternativa de suministro de propante en un área de explotación no convencional que minimice los costos logísticos asociados a esta, se desarrollará una metodología basada en evaluación de alternativas de igual servicio, que de acuerdo con Varela (2010) son aquellas en las cuales los beneficios generados por cada alternativa son exactamente los mismos, por lo cual la comparación se realiza en función de los costos o egresos que genera. Además teniendo en cuenta que algunas variables son aleatorias se utiliza una herramienta de simulación para evaluar las diferentes alternativas. A continuación se describe la metodología empleada.

\subsection{DEFINIR LAS ALTERNATIVAS} Y LAS RESPECTIVAS VARIABLES $Y$ PARÁMETROS A TENER EN CUENTA PARA SU EVALUACIÓN.

Alternativa 1: Evaluar el suministro de agentes de sostén a partir de una Compañía de Servicio Técnico, que cubra el costo del propante, el almacenamiento, la administración y el bombeo. Mediante la ecuación (1) se estimará el costo total anual de esta alternativa (los parámetros de la ecuación se definen en la tabla 1).

$$
\mathrm{C}_{1}=\left(\mathrm{CU}_{\text {có a }} * \mathrm{NE} * \mathrm{NL} * \mathrm{NP}\right)+\mathrm{CTN}+\mathrm{CTD}
$$

Alternativa 2: Evaluar la opción de comprar a un intermediario importador del producto en Colombia. Esta opción cubre el costo desde el fabricante en China, incluyendo los costos relacionados con los trámites de importación. La empresa operadora cubre los costos asociados al transporte nacional, mantener y administrar el inventario de apuntalante y por último el bombeo del propante. De esta alternativa se derivan dos opciones que estarían dadas si el propante es almacenado por la empresa operadora o por el intermediario. Mediante la ecuación (2) se estimará el costo total anual de esta alternativa

$$
\begin{gathered}
\mathrm{C}_{2}=\left(\mathrm{CU}_{\text {I cóa }} * \mathrm{NE} * \mathrm{NL} * \mathrm{NP}\right)+\mathrm{CB}+ \\
\mathrm{CTN}+\mathrm{CI}+\mathrm{CA}
\end{gathered}
$$

Alternativa 3: Evaluar la opción de que la empresa operadora realice la importación directamente con los fabricantes desde China hasta el pozo en situ. En este caso la empresa se ocuparía de todos los costos asociados al proceso de importación desde que se lo entregan en puerto chino hasta que lo nacionaliza en Colombia, así como los costos relacionados con el transporte nacional, el almacenamiento, el mantenimiento y la administración del inventario de propante y el bombeo en pozo. Mediante la ecuación (3) se estimará el costo total anual de esta alternativa

$$
\begin{aligned}
\mathrm{C}_{3}= & \left(\mathrm{CU}_{\mathrm{FOB} \text { có } \mathrm{a}} * \mathrm{NE} * \mathrm{NL} * \mathrm{NP}\right)+\mathrm{CB}+ \\
& \mathrm{CTN}+\mathrm{CTI}+\mathrm{COP}+\mathrm{CI}+\mathrm{CA}
\end{aligned}
$$

En la Tabla 1 se describen las variables aleatorias y los parámetros utilizados en las ecuaciones 1,2

\begin{tabular}{|c|c|c|}
\hline Variable & Definición & Tipo \\
\hline $\begin{array}{l}\mathrm{CU}_{\mathrm{C}} \\
\mathrm{CU}_{\mathrm{a}}\end{array}$ & $\begin{array}{l}\text { Costo unitario por libra de propante ofrecido } \\
\text { por la CST (arena y cerámico). }\end{array}$ & $\mathrm{P}$ \\
\hline $\begin{array}{l}\mathrm{CU}_{\text {IC }} \\
\mathrm{CU}_{\text {Ia }}\end{array}$ & $\begin{array}{l}\text { Costo unitario por libra de propante ofrecido } \\
\text { por el Intermediario (arena y cerámico). }\end{array}$ & $\mathrm{P}$ \\
\hline $\begin{array}{l}\mathrm{CU}_{\mathrm{FOB}-\mathrm{C}} \\
\mathrm{CU}_{\mathrm{FOB}-\mathrm{a}}\end{array}$ & $\begin{array}{l}\text { Costo unitario por libra Free on Board en } \\
\text { China (arena y cerámico). }\end{array}$ & $\mathrm{P}$ \\
\hline NE & Número de etapas por pozo. & $\mathrm{P}$ \\
\hline NL & Número de libras consumidas por etapa. & $\mathrm{P}$ \\
\hline NP & Número de pozos a explotar por año. & $\mathrm{D}$ \\
\hline CTN & Costo de transporte terrestre en Colombia. & $\mathrm{D}$ \\
\hline CTD & $\begin{array}{l}\text { Costo de transporte terrestre en Colombia por } \\
\text { devolución, este costo se presenta cuando la } \\
\text { empresa operadora debe regresar propante a la } \\
\text { CST, debido a un exceso de pedido. }\end{array}$ & $\mathrm{D}$ \\
\hline $\mathrm{CB}$ & $\begin{array}{l}\text { Costo total por bombeo. Su cálculo integra el } \\
\text { costo por bombeo por etapa y el costo por el } \\
\text { set de bombeo por pozo. }\end{array}$ & $\mathrm{D}$ \\
\hline $\mathrm{CI}$ & Costo de mantener inventario. & $\mathrm{D}$ \\
\hline CA & $\begin{array}{l}\text { Costo administrativo. Se calcula a través } \\
\text { de la suma de los costos correspondientes } \\
\text { a almacenamiento en bodega en } \\
\text { Barrancabermeja, vigilancia, administrador } \\
\text { de la bodega, operario de montacargas, } \\
\text { montacargas y transporte desde la bodega al } \\
\text { pozo. }\end{array}$ & $\mathrm{D}$ \\
\hline CTI & $\begin{array}{l}\text { Costo de transporte desde el Puerto en China } \\
\text { hasta el puerto en Colombia. }\end{array}$ & $\mathrm{D}$ \\
\hline $\mathrm{COP}$ & $\begin{array}{l}\text { Costo de operaciones portuarias en Colombia. } \\
\text { Este cálculo incluye los costos asociados } \\
\text { a seguro de mercancía, preparación de } \\
\text { documentos, autorización de aduana y control } \\
\text { técnico, manejo en terminal y gravamen } \\
\text { arancelario. }\end{array}$ & $\mathrm{D}$ \\
\hline
\end{tabular}
y 3, estableciendo en la columna tipo su respectiva condición, probabilístico (P) o determinístico (D).

Tabla 1. Variables y parámetros de las alternativas seleccionadas.

Fuente: elaboración propia. "P" Probabilístico por ser variable; "D" Determinístico por ser un parámetro. Adicional a lo anterior se toma como aleatoria la tasa de cambio del peso frente al dólar. 
4.2 ESPECIFICAR LOS DATOS DETERMINÍSTICOS Y PROBABILÍSTICOS DE LAS VARIABLES DE ENTRADA A PARTIR DE DATOS PROYECTADOS DE UN ÁREA CON CARACTERÍSTICAS SIMILARES AL ÁREA A EXPLOTAR; COTIZACIONES DE PROVEEDORES; JUICIOS DE EXPERTOS; ARTIÍCULOS E INFORMES TÉCNICOS.

A continuación se expone el origen de los datos utilizados en las diferentes variables y parámetros de las tres alternativas planteadas.

\subsubsection{Variables Aleatorias}

En la Tabla 2 se presenta un resumen de las nueve variables aleatorias junto con la distribución de probabilidad teórica que mejor describe su comportamiento, los parámetros asociados a cada una de las distribuciones y la fuente de donde se obtuvieron los datos.

Considerando que algunas distribuciones de las variables aleatorias pueden tomar valores negativos, se hizo necesario establecer límites de truncamiento para evitar resultados inconsistentes con el objetivo de esta investigación. Estos límites se establecieron multiplicando la media de la distribución más tres desviaciones estándar (límite superior) y menos tres desviaciones estándar (límite inferior), esto considerando que de acuerdo con el teorema de Chebyshev el porcentaje de los datos que debe caer dentro de 3 desviaciones estándar para una distribución en forma de campana es alrededor de 99,7\% (Freund \& Simon, 1994).

Tabla 2. Distribución de probabilidad de las variables de entrada.

\begin{tabular}{|c|c|c|c|c|}
\hline Variable & Descripción & Distribución & Parámetros & Fuente \\
\hline Variable 1 & $\begin{array}{l}\text { Costo propante por libra tipo } \\
\text { arena (CST) }\end{array}$ & Normal & $\mu=0,6 ; \sigma=0.0067$ & $\begin{array}{l}\text { Media por juicio de expertos y desviación } \\
\text { estándar por variabilidad de los datos de arena } \\
\text { del Intermediario. }\end{array}$ \\
\hline Variable 2 & $\begin{array}{l}\text { Costo propante por libra tipo } \\
\text { cerámico (CST) }\end{array}$ & Normal & $\begin{array}{l}\mu=1,095 \\
\sigma=0,12426\end{array}$ & $\begin{array}{l}\text { Informe Técnico (Castillo \& Hernández, 2011, } \\
\text { pág. 24) }\end{array}$ \\
\hline Variable 3 & $\begin{array}{l}\text { Costo propante por libra tipo } \\
\text { arena (Intermediario) }\end{array}$ & Normal & $\mu=0,4 ; \sigma=0.0067$ & Juicio de Expertos \\
\hline Variable 4 & $\begin{array}{l}\text { Costo propante por libra tipo } \\
\text { cerámico (Intermediario) }\end{array}$ & Normal & $\mu=0,8 ; \sigma=0,12426$ & $\begin{array}{l}\text { Media por juicio de expertos y desviación } \\
\text { estándar por variabilidad de los datos de } \\
\text { cerámicos de la CST. }\end{array}$ \\
\hline Variable 5 & $\begin{array}{l}\text { Costo propante por libra tipo } \\
\text { arena (Importación China) }\end{array}$ & Normal & $\begin{array}{l}\mu=0,148929 ; \sigma= \\
0,054463\end{array}$ & $\begin{array}{l}\text { Catálogo de productos de los proveedores en } \\
\text { China (Alibaba Group). }\end{array}$ \\
\hline Variable 6 & $\begin{array}{l}\text { Costo propante por libra tipo } \\
\text { cerámico (Importación China) }\end{array}$ & Normal & $\begin{array}{l}\mu=0,203225 ; \sigma= \\
0,053335\end{array}$ & $\begin{array}{l}\text { Catálogo de productos de los proveedores en } \\
\text { China (Alibaba Group). }\end{array}$ \\
\hline Variable 7 & Número de etapas por pozo. & $\begin{array}{l}\text { Uniforme } \\
\text { discreta }\end{array}$ & Min=4; Máx=16 & $\begin{array}{l}\text { Reservorio de Vaca Muerta (Instituto de Energía, } \\
\text { 2014) }\end{array}$ \\
\hline Variable 8 & $\begin{array}{l}\text { Número de libras de propante por } \\
\text { etapa. }\end{array}$ & Lognorm & $\begin{array}{l}\mu=482747,1 \\
\sigma=25039,8\end{array}$ & $\begin{array}{l}\text { Reservorio de Vaca Muerta (Instituto de Energía, } \\
\text { 2014) }\end{array}$ \\
\hline Variable 9 & Precio del dólar en el último año. & Normal & $\begin{array}{l}\mu=2408,91 \\
\sigma=277,54\end{array}$ & $\begin{array}{l}\text { Publicados en el último año por el Banco de la } \\
\text { Republica. }\end{array}$ \\
\hline
\end{tabular}

Fuente: elaboración propia con el software@Risk. Datos ajustados con el estadístico Anderson-Darling.

\subsubsection{PARÁMETROS}

Los datos utilizados para modelar las variables determinísticas se obtienen de varias fuentes: el número de pozos a explotar cada año es determinado por juicio de expertos; el costo de transporte terrestre en Colombia se extrae de Nodo Logístico (2010) y varía dependiendo del origen de la carga; el costo de bombeo por etapa y el costo por el set de bombeo por pozo se tomó de Sáchica \& Amaya (2010); el costo de mantener inventario, equivale a multiplicar el costo de capital de Ecopetrol del 12\% (Serrano, 2011, pág. 99) sobre la inversión del inventario medio; el costo de almacenamiento en Barrancabermeja se obtiene a partir del precio en el mercado del arrendamiento de bodegas en la zona; para la vigilancia se tiene en cuenta tres turnos de 8 horas tomando como referencia los parámetros de la Superintendencia de Vigilancia y Seguridad Privada (2014); la remuneración del administrador de la bodega se determina mediante la tabla de niveles salariales de carrera técnica y administrativa de Ecopetrol; el 
salario del operador de montacargas y del coordinador de compras e importaciones equivalen al promedio de sueldos en el mercado para este tipo de cargos; el costo de montacargas se asume como un leasing a diez años; lo relacionado con los costos del transporte de contenedores en barco se extraen del Sistema de Información Comercial de Proexport Colombia (2013); el seguro de la mercancía se cotiza con el Grupo ISCE (2015), en un $1 \%$ del valor de la carga; el gravamen arancelario corresponde al de arenas naturales de cualquier clase, tomado de la página de la Dirección de Impuestos y Aduanas Nacionales (2015); y por último, en lo que respecta a los costos relacionados con operaciones portuarias se toman del estudio Doing Business para la economía Colombiana, publicado por el Banco Mundial (2013).

\subsection{SiMULACIÓN POR MONTE CARLO (SOFTWARE @RISK).}

Para desarrollar la simulación se consideraron diferentes niveles de producción anuales $(10,30,50$, 70 y 100 pozos) tanto para los propantes tipo arena como cerámicos. Además, se planteó un descuento por volumen de compra de propante, el cual se presenta en la tabla 3.

Tabla 3. Descuento por volumen de compra de propante

\begin{tabular}{|ccc|}
\hline \multicolumn{2}{|c|}{ Rango en libras } & Descuento (\%) \\
\hline Min. & Máx. & 3 \\
\hline 44.092 .452 & 110.231 .131 & 5 \\
110.231 .131 & 176.369 .810 & 7 \\
\hline 176.369 .810 & 286.600 .941 & 10 \\
\hline 286.600 .941 & 352.739 .619 & 12 \\
\hline 352.739 .619 & $>$ & \\
\hline
\end{tabular}

\subsubsection{NÚMERO DE RÉPLICAS}

El número de réplicas hace referencia a la cantidad de simulaciones necesarias para encontrar resultados estadísticamente confiables. Para determinar el número de iteraciones de la simulación, se debe efectuar el cálculo de la varianza de un cierto número de iteraciones (premuestra), para esto se parte del supuesto que la distribución de esa probabilidad es normal; se define un nivel de confianza y con base en esta cifra se halla el valor $Z$ de la distribución normal. Además también debe determinarse el error que se está dispuesto a aceptar. La fórmula (Jiménez, Espinoza, \& Fonseca, 2007) se presenta a continuación:

$$
n=\frac{Z_{\alpha / 2}^{2} \sigma^{2}}{e^{2}}
$$

Donde,

$\mathrm{n}=$ número de iteraciones.

$\mathrm{Z}_{\alpha / 2}=$ estadístico de distribución normal.

$\alpha=$ nivel de confianza.

$\sigma=$ desviación estándar de la premuestra.

$\mathrm{e}=$ error absoluto de estimación, depende del investigador.

Finalmente para hallar el número de iteraciones adecuado para la simulación, se hizo una corrida preliminar de 1000 veces, obteniendo una media y desviación estándar determinada, que luego se sustituyó en la ecuación (4) considerando un error del $1 \%$ y un $1.96 \mathrm{Z}_{\alpha / 2}$ para un nivel de significancia $\alpha=5 \%$, obteniendo como resultado un número de iteraciones $\mathrm{n}$ alrededor de las 10.000 .

\section{APLICACIÓN Y ANÁLISIS DE RESULTADOS}

En las Tablas 4, 5 y 6 se presentan las estructuras de costos propuestas para simular las tres alternativas de suministro de propantes. Los resultados presentados en cada una de las tablas son anuales y corresponden a ejecutar una corrida de simulación para un nivel de producción de diez pozos.

En la Tabla 4 se estiman los tres costos más notables, que corresponden a: costo del propante requerido estimado a partir de las variables cantidad (lb) y costo de propante por libra; costo por transporte; y el costo por devolución que se estima a partir de la probabilidad de ocurrencia de un consumo inferior al estimado inicialmente. Al realizar un análisis vertical de cada uno de los costos, se observa que el más relevante es el costo del propante requerido con un $98,739 \%$ y un $97,719 \%$ para cerámico y arena respectivamente, mientras que el costo por devolución es prácticamente nulo.

Es importante destacar que el costo del propante por libra en la Tabla 4 incluye los costos por bombeo, administración e inventario, por tanto en la Tabla 5 estos se incluyen como marginales complementado su estructura. Adicional a lo anterior, se presenta el costo total considerando que el intermediario entrega el producto en su base en Barrancabermeja, en el cual la empresa operadora se ahorraría los costos por transporte, administración e inventario. En el análisis vertical se observa que considerando la opción de almacenar o no el costo más representativo es el del propante requerido, mientras que el costo de menor porcentaje de participación es el transporte al pozo y el de inventario para la opción sin almacenamiento y con almacenamiento respectivamente. 
Tabla 4. Estructura de costos mediante una CST (Alternativa 1)

\begin{tabular}{|c|c|c|c|c|}
\hline Ítem & Cerámico & $\%$ & Arena & $\%$ \\
\hline Cantidad de propante por etapa (Lb) & $482.747,10$ & & $482.747,10$ & \\
\hline No. de etapas de Frac/Pozo & 10 & & 10 & \\
\hline Cantidad de propante Requerido (Lb/Pozo) & $4^{\prime} 827.471$ & & $4^{\prime} 827.471$ & \\
\hline Costo de propante (USD/Lb) & 1,1 & & 0,6 & \\
\hline Costo total de propante por pozo (USD) & $5 ’ 291.657,39$ & & 2’896.482,60 & \\
\hline No. de Pozos & 10 & & 10 & \\
\hline Total Cantidad de Propante Requerido (Lb) & $48^{\prime} 274.710$ & & $48^{\prime} 274.710$ & \\
\hline Costo del Propante Requerido (USD) & $51 ' 858.242$ & 98,713 & $28^{\prime} 385.529$ & 97,674 \\
\hline Total Cantidad de Propante Requerido (ton) & $21^{\prime} 897,04$ & & $21^{\prime} 897,04$ & \\
\hline Costo Viaje Bogotá-Barranca (USD/ton) & 30,87 & & 30,87 & \\
\hline Costo por Transporte (USD) & 676.034 & 1,287 & 676.034 & 2,326 \\
\hline Cantidad de propante devuelto (ton) & 0,58 & & 0,58 & \\
\hline Costo por Devolución (USD) & 18 & 0,00003 & 18 & 0,0001 \\
\hline COSTO TOTAL ANUAL (USD) & $52,534.294,09$ & 100 & 29’061.581,13 & 100 \\
\hline
\end{tabular}

Fuente: elaboración propia. La diferencia en unidades de toneladas y libras se debe a que el costo unitario suministrado por el respectivo proveedor así lo exige. Una tonelada equivale a 2204,62 libras.

Tabla 5. Estructura de costos mediante un Intermediario (Alternativa 2)

\begin{tabular}{|c|c|c|c|c|c|}
\hline Ítem & & Cerámico & $\%$ & Arena & $\%$ \\
\hline Cantidad de propante por etapa $(\mathrm{Lb})$ & & 482.747 & & 482.747 & \\
\hline No. de etapas de Frac/Pozo & & 10 & & 10 & \\
\hline Cantidad de propante Requerido (Lb/Pozo) & & $4^{\prime} 827.471$ & & $4^{\prime} 827.471$ & \\
\hline Costo de Propante (USD/Lb) & & 0,8 & & 0,4 & \\
\hline Costo total de propante por pozo (USD) & & 3'861.977 & & 1'930.988 & \\
\hline No. de Pozos & & 10 & & 10 & \\
\hline Total Cantidad de Propante Requerido (Lb) & & $48^{\prime} 274.710$ & & $48^{\prime} 274.710$ & \\
\hline Costo del Propante Requerido (USD) & & $37^{\prime} 461.175$ & $98,47 / 96,302$ & $18^{\prime} 730.587$ & $96,99 / 93,091$ \\
\hline Total Cantidad de Propante Requerido (ton) & & $21^{\prime} 897,04$ & & $21^{\prime} 897,04$ & \\
\hline Costo Viaje Bogotá-Barranca (USD/ton) & & 30,87 & & 30,87 & \\
\hline Costo por Transporte (USD) & & 676.034 & $0 / 1,738$ & 676.034 & $0 / 3,360$ \\
\hline Costo por bombeo/etapa (USD) & 3.000 & 300.000 & & 300.000 & \\
\hline Costo por Set de bombeo/pozo (USD) & 8.000 & 80.000 & & 80.000 & \\
\hline Costo por Bombeo (USD) & & 380.000 & $1,00 / 0,977$ & 380.000 & $1,97 / 1,889$ \\
\hline Tamaño de pedido cada 15 días (ton) & & 912,4 & & 912,4 & \\
\hline Arrendamiento bodega (USD) & & $35.155,8$ & & $35.155,8$ & \\
\hline Vigilancia (turnos) (USD) & 3 & 31.071 & & 31.071 & \\
\hline Administrador de la bodega (USD) & 1 & 11.546 & & 11.546 & \\
\hline Operario de montacargas (USD) & 1 & 3.487 & & 3.487 & \\
\hline Montacargas (USD) & 1 & 2.500 & & 2.500 & \\
\hline Transporte al pozo (USD) & & 202.000 & $0,53 / 0$ & 202.000 & $1,05 / 0$ \\
\hline Costo Administrativo (USD) & & $285.760,14$ & $0 / 0,735$ & $285.760,14$ & $0 / 1,42$ \\
\hline Costo financiero (USD) & $12 \%$ & $96.549,4$ & & $48.274,7$ & \\
\hline Costo por Inventario (USD) & & $96.549,42$ & $0 / 0,248$ & $48.274,71$ & $0 / 0,24$ \\
\hline $\begin{array}{l}\text { COSTO TOTAL ANUAL } \\
\text { SIN ALMACENAMIENTO(USD) }\end{array}$ & & $38^{\prime} 043.175,40$ & 100 & $19^{\prime} 312.587,92$ & 100 \\
\hline $\begin{array}{l}\text { COSTO TOTAL ANUAL } \\
\text { CON ALMACENAMIENTO(USD) }\end{array}$ & & $38 ' 899.518,39$ & 100 & $20 ’ 120.656,20$ & 100 \\
\hline
\end{tabular}

Fuente: elaboración propia. El símbolo “/” separa el porcentaje de participación de la opción sin y con almacenamiento. La diferencia en unidades de toneladas y libras se debe a que el costo unitario suministrado por el respectivo proveedor así lo exige. Una tonelada equivale a 2204,62 libras. 
En la Tabla 6 se estiman principalmente los costos de propante requerido, transporte terrestre, bombeo, administración, inventario y operaciones portuarias que componen la opción de que la empresa operadora realice el proceso de suministro directamente. De acuerdo con el análisis vertical (ver tabla 6), se resalta que los costos más sobresalientes son los causados por el propante requerido, las operaciones portuarias y el transporte terrestre, alcanzando un porcentaje entre los tres del 94,52 y el 93,04 para apuntalante cerámico y arena respectivamente; asimismo se observa que el costo por inventario es el menos representativo de todos.

Tabla 6. Estructura de costos mediante importación directa (Alternativa 3)

\begin{tabular}{|c|c|c|c|c|c|}
\hline Ítem & & Cerámico & $\%$ & Arena & $\%$ \\
\hline Cantidad de propante por etapa (Lb) & & 482.747 & & 482.747 & \\
\hline No. De etapas de Frac/Pozo Horizontal & & 10 & & 10 & \\
\hline Cantidad de propante Requerido ( $\mathrm{Lb} / \mathrm{Pozo})$ & & $4^{\prime} 827.471$ & & $4^{\prime} 827.471$ & \\
\hline Costo de Propante FOB (USD/Lb) & & 0,21 & & 0,15 & \\
\hline Costo total de propante por pozo (USD) & & $1^{\prime} 008.031$ & & 732.637 & \\
\hline No. De Pozos & & 10 & & 10 & \\
\hline Total Cantidad de Propante Requerido (Lb) & & $48^{\prime} 274.710$ & & $48^{\prime} 274.710$ & \\
\hline Costo del Propante Requerido (USD) & & 9'777.900 & 76,14 & 7'106.577 & 71,04 \\
\hline Total Cantidad de Propante Requerido (ton) & & $21^{\prime} 897,04$ & & $21^{\prime} 897,04$ & \\
\hline Costo Viaje Cartagena-Barranca (USD/ton) & & 45,82 & & 45,82 & \\
\hline Costo por Transporte (USD) & & 1'003.411 & 7,81 & 1'003.411 & 10,03 \\
\hline Costo por bombeo/etapa (USD) & 3.000 & 300.000 & & 300.000 & \\
\hline Costo por Set de bombeo/pozo (USD) & 8.000 & 80.000 & & 80.000 & \\
\hline Costo por Bombeo (USD) & & 380.000 & 2,96 & 380.000 & 3,80 \\
\hline Tamaño de pedido (ton) cada 15 días & & 912,4 & & 912,4 & \\
\hline Arrendamiento bodega (USD) & & $35.155,8$ & & $35.155,8$ & \\
\hline Vigilancia (turnos) (USD) & 3 & 31.071 & & 31.071 & \\
\hline Administrador de la bodega (USD) & 1 & 11.546 & & 11.546 & \\
\hline Coordinación de compras e importación (USD) & 1 & 12.454 & & 12.454 & \\
\hline Operario de montacargas (USD) & 1 & 3.487 & & 3.487 & \\
\hline Montacargas (USD) & 1 & 2.500 & & 2.500 & \\
\hline Transporte al pozo (USD) & & 202.000 & & 202.000 & \\
\hline Costo Administrativo (USD) & & $298.213,90$ & 2,32 & $298.213,90$ & 2,98 \\
\hline Costo financiero (USD) & $12 \%$ & $25.200,8$ & & $18.315,9$ & \\
\hline Costo por Inventario (USD) & & $25.200,77$ & 0,20 & $18.315,92$ & 0,18 \\
\hline $\begin{array}{l}\text { Transporte principal Barco China-Colombia } \\
\text { Contenedor de 20' (USD) }\end{array}$ & 1170 & 517.995 & & 517.995 & \\
\hline Seguro de mercancías (USD) & $1 \%$ & 97.779 & & 71.066 & \\
\hline Preparación de documentos (USD) & 250 & 110.683 & & 110.683 & \\
\hline Autorización de aduana y control técnico (USD) & 170 & 75.264 & & 75.264 & \\
\hline Puertos y manejo terminal (USD) & 150 & 66.410 & & 66.410 & \\
\hline Gravamen arancelario (USD) & $5 \%$ & $488.895,0$ & & $355.328,9$ & \\
\hline Costo de Operaciones Portuarias (USD) & & 1’357.025,04 & 10,57 & 1'196.745,69 & 11,96 \\
\hline COSTO TOTAL ANUAL (USD) & & $12{ }^{\prime} 841.750,44$ & 100 & $10^{\prime} 003.264$ & 100 \\
\hline
\end{tabular}

Fuente: elaboración propia. La diferencia en unidades de toneladas y libras se debe a que el costo unitario suministrado por el respectivo proveedor así lo exige. Una tonelada equivale a 2204,62 libras. 
Teniendo en cuenta que los costos totales de la alternativa 2 con y sin almacenamiento son muy similares, se decidió realizar la simulación con la alternativa 2 sin almacenamiento. Los resultados de la media, desviación estándar e intervalos a un nivel de confianza del 90\%
(IC) de las corridas del modelo para las tres alternativas planteadas se presentan en la tabla 7. Adicionalmente en las Figuras $\mathbf{1}$ y $\mathbf{2}$ se exponen las gráficas de la media y la desviación estándar.

En la Tabla 7 se evidencia que los intervalos de confianza

Tabla 7. Resultados simulación

\begin{tabular}{|c|c|c|c|c|c|c|c|}
\hline \multirow{2}{*}{$\mathbf{N}^{\circ}$ de pozos } & \multirow{2}{*}{ Estadístico } & \multicolumn{2}{|c|}{ CST } & \multicolumn{2}{|c|}{ INTERMEDIARIO } & \multicolumn{2}{|c|}{ IMPORTACIÓN DIRECTA } \\
\hline & & Cerámico & Arena & Cerámico & Arena & Cerámico & Arena \\
\hline \multirow{4}{*}{$\begin{array}{l}\text { on } \\
\text { N } \\
\stackrel{2}{0} \\
\stackrel{0}{0}\end{array}$} & Media & $52.454 .771,7$ & $29.029 .410,4$ & $38.368 .954,2$ & $19.475 .943,6$ & $12.939 .490,5$ & $10.083 .877,5$ \\
\hline & Desv. Est & $20.198 .476,6$ & $10.689 .764,3$ & $15.398 .501,2$ & $7.147 .888,7$ & $5.152 .977,2$ & $4.167 .460,7$ \\
\hline & \multirow{2}{*}{ IC $90 \%$} & $22.135 .610,0$ & $12.068 .000,0$ & $15.791 .500,0$ & $8.140 .950,0$ & $5.402 .147,0$ & $4.043 .574,0$ \\
\hline & & $86.167 .560,0$ & $45.795 .960,0$ & $64.715 .420,0$ & $30.758 .440,0$ & $21.873 .730,0$ & $17.587 .670,0$ \\
\hline \multirow{4}{*}{ 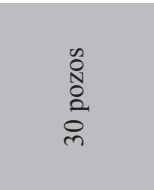 } & Media & $152.001 .964,1$ & $84.131 .812,5$ & $111.142 .479,5$ & $56.455 .234,2$ & $37.626 .004,7$ & $29.366 .792,0$ \\
\hline & Desv. Est & $58.128 .087,4$ & $30.746 .103,9$ & $44.230 .689,8$ & $20.555 .516,7$ & $14.985 .675,5$ & $12.216 .036,9$ \\
\hline & \multirow{2}{*}{ IC $90 \%$} & $63.401 .950,0$ & $35.151 .330,0$ & $45.996 .020,0$ & $23.723 .760,0$ & $15.610 .140,0$ & $11.903 .670,0$ \\
\hline & & $247.985 .100,0$ & $132.088 .800,0$ & $187.427 .800,0$ & $88.540 .460,0$ & $63.362 .390,0$ & $50.944 .370,0$ \\
\hline \multirow{4}{*}{$\begin{array}{l}n \\
\stackrel{n}{N} \\
\stackrel{2}{0} \\
\stackrel{2}{n}\end{array}$} & Media & $245.260 .304,4$ & $135.856 .654,5$ & $179.449 .643,0$ & $91.170 .497,1$ & $60.739 .800,6$ & $47.477 .232,9$ \\
\hline & Desv. Est & $90.651 .943,7$ & $48.029 .585,4$ & $69.324 .192,1$ & $32.090 .658,3$ & $23.464 .407,9$ & $19.135 .859,2$ \\
\hline & \multirow{2}{*}{ IC $90 \%$} & $106.487 .200,0$ & $58.987 .800,0$ & $76.190 .120,0$ & $39.785 .730,0$ & $26.047 .090,0$ & $19.524 .670,0$ \\
\hline & & $392.152 .100,0$ & $207.841 .900,0$ & $293.677 .600,0$ & $139.405 .600,0$ & $101.269 .400,0$ & $81.532 .980,0$ \\
\hline \multirow{4}{*}{ 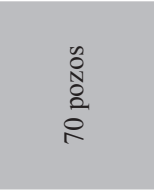 } & Media & $336.773 .550,8$ & $186.500 .473,9$ & $246.454 .865,0$ & $125.197 .040,0$ & $83.873 .287,1$ & $65.532 .867,3$ \\
\hline & Desv. Est & $126.450 .847,2$ & $66.726 .397,3$ & $96.673 .072,6$ & $44.633 .326,3$ & $33.072 .374,4$ & $26.569 .484,3$ \\
\hline & \multirow{2}{*}{ IC $90 \%$} & $145.260 .700,0$ & $80.210 .740,0$ & $104.160 .200,0$ & $54.110 .910,0$ & $35.782 .810,0$ & $27.319 .020,0$ \\
\hline & & $549.710 .700,0$ & $292.557 .900,0$ & $412.692 .100,0$ & $195.537 .000,0$ & $143.166 .700,0$ & $113.516 .600,0$ \\
\hline \multirow{4}{*}{$\begin{array}{l}\text { o } \\
\text { o } \\
\stackrel{2}{0} \\
\stackrel{0}{0}\end{array}$} & Media & $476.076 .424,7$ & $263.639 .636,3$ & $348.003 .852,6$ & $176.968 .884,1$ & $118.933 .266,4$ & $93.085 .360,2$ \\
\hline & Desv. Est & $183.761 .394,9$ & $97.212 .146,6$ & $139.667 .126,2$ & $64.962 .135,2$ & $47.831 .763,1$ & $38.859 .761,9$ \\
\hline & \multirow{2}{*}{ IC $90 \%$} & $204.279 .000,0$ & $112.321 .900,0$ & $146.608 .500,0$ & $75.842 .660,0$ & $50.226 .250,0$ & $38.051 .810,0$ \\
\hline & & $784.623 .400,0$ & $416.596 .000,0$ & $589.319 .900,0$ & $279.850 .000,0$ & $202.794 .300,0$ & $162.278 .400,0$ \\
\hline
\end{tabular}

Fuente: elaboración propia con resultados del software@Risk.

se traslapan; sin embargo después de realizar una prueba ANOVA (análisis de varianza) para contrastar la hipótesis nula de que las medias de las tres alternativas son iguales, frente a la hipótesis alternativa de que por lo menos una de las medias de las tres alternativas es diferente, se halló que las diferencias entre las medias de las alternativas es significativa, lo que indica que existe gran variabilidad entre las opciones de suministro de propantes planteadas.

En la Figura 1 se puede observar que la media es más alta para los apuntalantes tipo cerámico y tipo arena de la alternativa de suministro a través de una CST, mientras que los valores más bajos de este estadístico los presenta la alternativa de importación directa desde China; así mismo al analizar la desviación estándar (Figura 2) se encuentra que la dispersión de los datos presenta un comportamiento similar a la media con un aumento a medida que los niveles de producción crecen, esto último indica que en la alternativa 3 (importación directa) existe menos riesgo de obtener valores muy alejados del promedio. Además se advierte que la opción de comprar cerámicos es mejor para la alternativa de importación directa, dado que las diferencias en costo medio con la arena son más pequeñas respecto de las otras dos alternativas. 


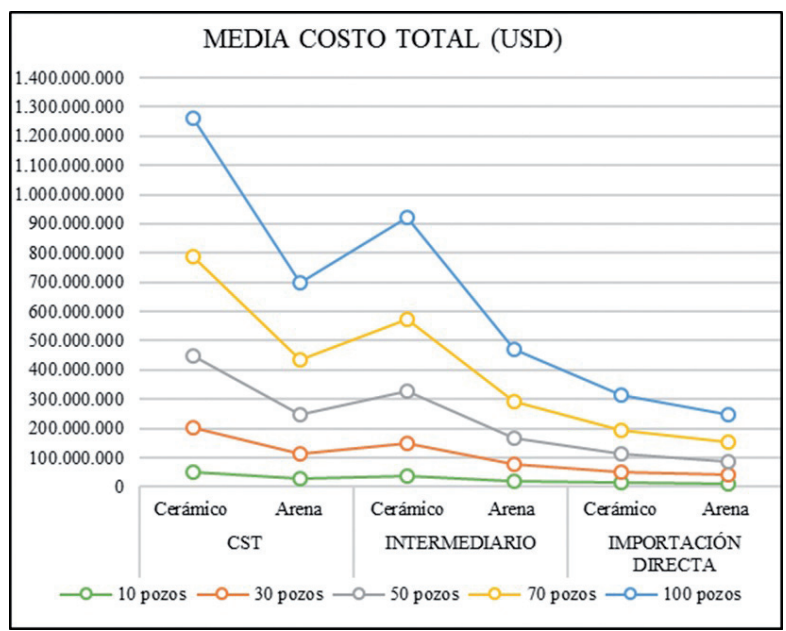

Figura 1. Media del Costo Total de la simulación

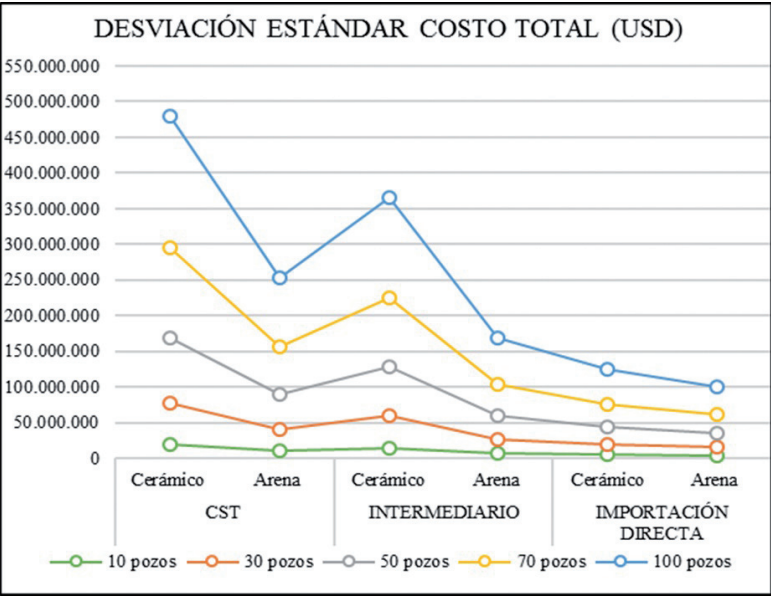

Figura 2. Desviación Estándar del Costo Total de la simulación

En la Figura 3 se expone el cambio en el costo unitario del propante para las tres alternativas seleccionadas, para diferentes niveles de producción. En esta se observa que el costo por libra de la alternativa de suministro a través de una CST es en promedio 1,37 veces y 1,49 veces el costo por libra de comprar propante tipo cerámico y arena con un intermediario respectivamente; así mismo al compararlo con el precio del propante por libra de la importación directa se aprecia que este equivale en promedio a 3 veces el costo del cerámico y a 1,87 veces el costo de la arena. Además, si se adquiere con un Intermediario saldría en promedio 1,96 y 0,92 veces más caro que comprarlo por medio de la importación directa. Finalmente, tal como se analizó en la Figura 1 la diferencia entre el costo unitario del cerámico y la arena es relativamente pequeña, lo que sugiere que al importar directamente sería mejor la opción de traer cerámicos, teniendo en cuenta las propiedades de fractura de estos.

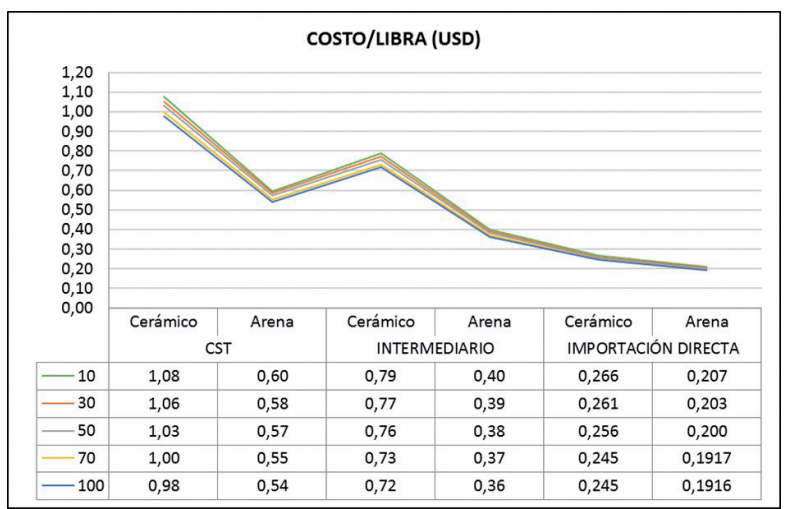

Figura 3. Costo Unitario

Teniendo en cuenta que los posibles proveedores de estas materias primas no ofrecen información sobre descuentos por volúmenes, fue necesario hacer estimaciones para varios rangos de descuentos, en los cuales se obtuvo que la diferencia de costos para las tres alternativas sigue siendo consistente con los resultados presentados anteriormente.

En las gráficas de araña de un nivel de producción de diez pozos para agente de sostén tipo cerámico y tipo arena de cada una de las alternativas planteadas (ver Figuras $4,5,6,7,8$ y 9), se observa que la variable de entrada que mayor impacto tiene sobre el valor del costo total es el número de etapas por pozo. Sin embargo, la segunda variable de mayor influencia difiere dependiendo del tipo de propante, para cerámico es el costo por libra de material y para arena es el número de libras de propante por etapa (alternativa 1 y 2) y el costo por libra (alternativa 3). Además se evidencia que el costo total presenta menos sensibilidad a las variaciones en la tasa de cambio peso-dólar. También cabe resaltar que todas las variables de entrada tienen pendiente positiva lo que indica que guardan una relación directa con la variable respuesta. Iguales resultados se presentan para cantidades de producción superior.

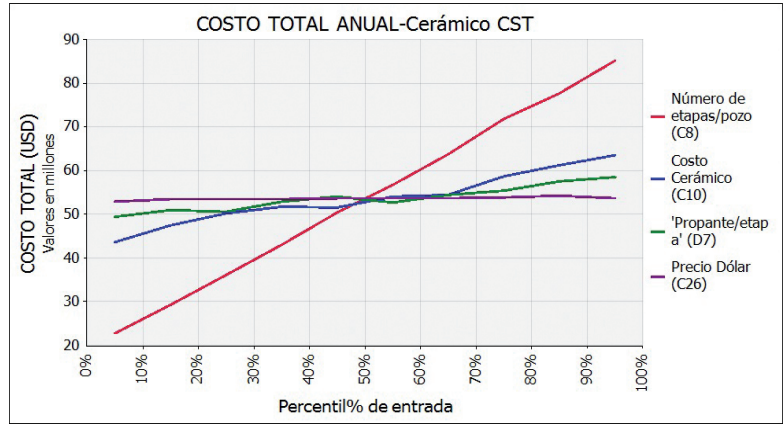

Figura 4. Gráfico de araña alternativa 1 (Cerámico) Fuente: elaboración propia en software@Risk 


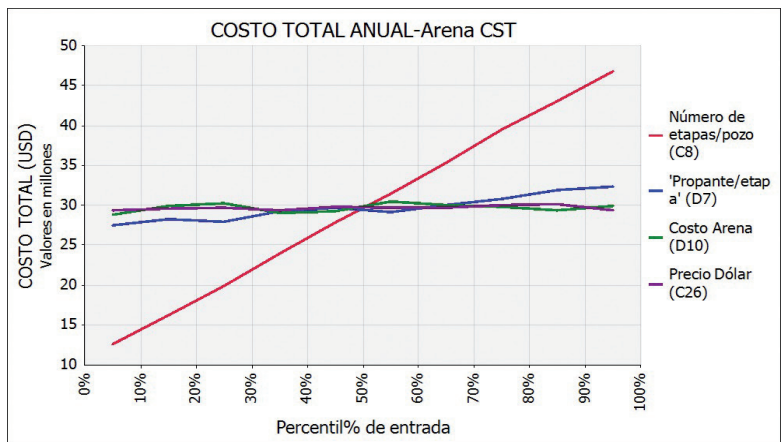

Figura 5. Gráfico de araña alternativa 1 (Arena) Fuente: elaboración propia en software@Risk

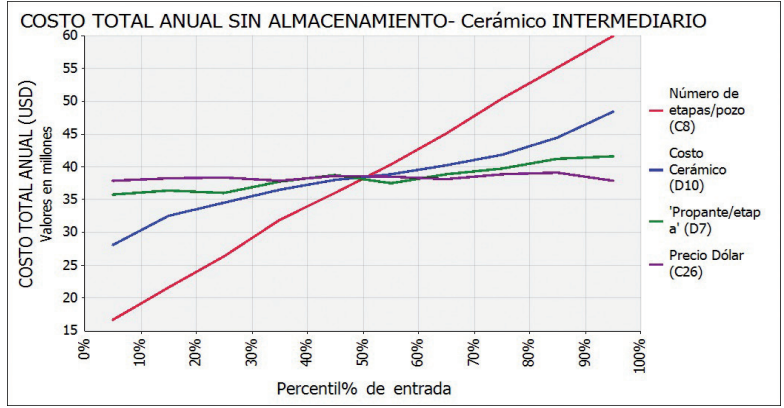

Figura 6. Gráfico de araña alternativa 2 (Cerámico)

Fuente: elaboración propia en software @ Risk

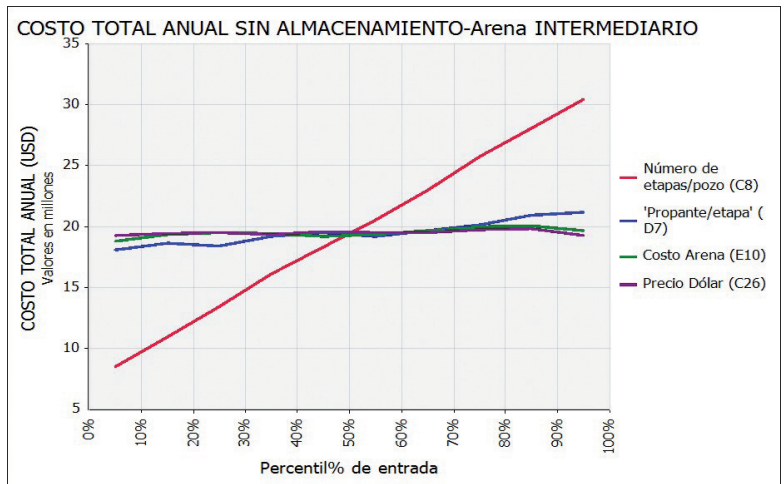

Figura 7. Gráfico de araña alternativa 2 (Arena)

Fuente: elaboración propia en software @Risk

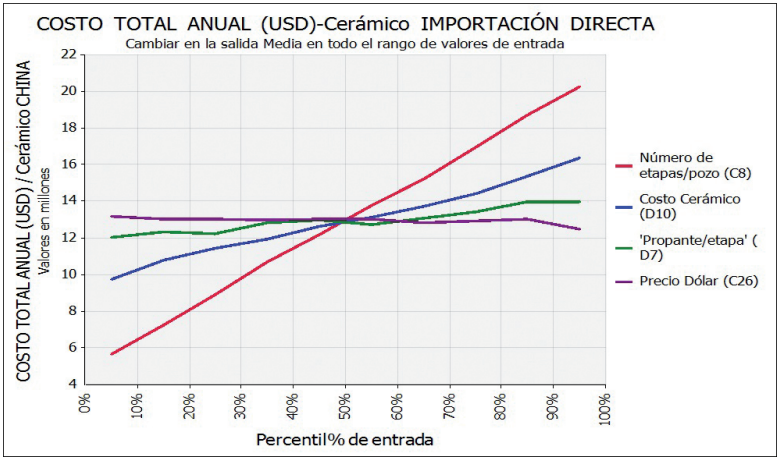

Figura 8. Gráfico de araña alternativa 3 (Cerámico) Fuente: elaboración propia en software@Risk

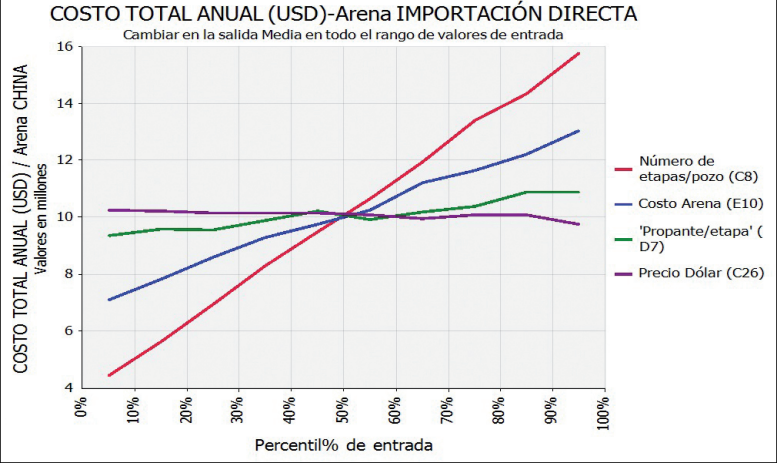

Figura 9. Gráfico de araña alternativa 3 (Arena)

Fuente: elaboración propia en software @Risk

\section{CONCLUSIONES Y RECOMENDACIONES}

- De los resultados de la presente investigación se podría inferir que bajo las condiciones y supuestos dados, la mejor alternativa para el suministro de propantes para un yacimiento no convencional en las inmediaciones del Valle del Magdalena Medio, es la alternativa de importación directa por parte de la empresa operadora, ya que su costo es 1,96 y 0.92 veces menos para apuntalante cerámico y arena respectivamente que para la alternativa de comprarlo con un Intermediario. Mientras que al comparar esta alternativa con la CST su costo es 3,04 y 1,87 veces menor para propante cerámico y arena respectivamente.

- $\mathrm{Al}$ analizar tanto los costos totales como unitarios de los propantes tipo cerámico y tipo arena para la alternativa de importación directa se observa que la diferencia entre ellos es relativamente baja, por tal motivo se recomendaría inclinarse por la adquisición de propante tipo cerámico teniendo en cuenta sus mejores propiedades de fractura sobre la arena.

- La evaluación de la simulación permitió identificar que la variable de entrada número de etapas por pozo es la que mayor impacto tiene sobre el cambio en la media de la variable Costo total para las tres alternativas planteadas.

- Teniendo en cuenta los resultados, en el caso de que la empresa operadora abastezca la materia prima a través de la empresa intermediadora, debería negociar que la ubicación de la bodega de almacenamiento desde la cual se realiza el suministro quedara en las inmediaciones de 
Barrancabermeja teniendo en cuenta los volúmenes de pedido. Esto con el fin de no incurrir en costos asociados al almacenamiento.

- Se recomienda completar la simulación teniendo en cuenta otros porcentajes de descuento por volumen de compra, considerando otras fuentes de abastecimiento e incluyendo la variable eficiencia de acuerdo al método de fracturamiento y el tipo de propante.

- En la alternativa 3 (importación directa) solo se tuvo en cuenta como proveedor del propante a un fabricante en China, debido a la escasa disponibilidad de datos proporcionados por proveedores en otros países.

- Los resultados de este trabajo de investigación deben ser tenidos en cuenta siempre y cuando se cumplan los supuestos, bajo los cuales se estimaron los costos totales, entre ellos los tipos de distribución, medias y varianzas de las diferentes variables estocásticas (precio unitario del propante, número de etapas por pozo, propante por etapa) además de los parámetros determinísticos (Costo de transporte, bombeo, almacenamiento, administración, entre otros). En el evento en que estos supuestos se modifiquen, el modelo presentado en el presente trabajo permite reestimar los costos de suministro de propante.

\section{BIBLIOGRAFÍA}

1. Aguirre, R. (16 de Noviembre de 2013). Clave de un insumo central en el mundo Shale. Obtenido de Diario Rio Negro: http://www.rionegro.com.ar/ diario/el-sosten-1377329-10948-notas_energia.aspx

2. Banco Mundial. (2013). Doing Business en Colombia 2013. Washington: Banco Mundial y la Corporación Financiera Internacional.

3. Barzola, G. (Agosto de 2012). La elección de la arena es una de las decisiones más importantes en la etapa exploratoria. (Petrotecnia, Entrevistador)

4. Caballero, S., \& Carrillo, B. H. (2012). Selección del material propante en procesos de fracturamiento hidráulico en un pozo petrolero. Bucaramanga: Universidad Industrial de Santander.
5. Castillo, R., \& Hernández, A. (2011). estudio básico de mercado para el suministro de propantes a nivel local, para yacimientos de Shale Gas. Piedecuesta: Ecopetrol-ICP.

6. Dirección de Impuestos y Aduanas Nacionales . (10 de Septiembre de 2015). DIAN-Muisca-Arancel. Obtenido de DIAN: https://muisca.dian.gov.co/ WebArancel/DefResultadoConsNomenclaturas.fac es;jsessionid=F600886327CEE017E0A703F23003 00B7

7. Freund, J. E., \& Simon, G. A. (1994). Estadísitca Elemental . Naucalpan de Juárez: Prentice Hall Hispanoamericana, S.A.

8. Grupo ISCE. (14 de Septiembre de 2015). Servicios: Seguro de Carga. Obtenido de Grupo ISCE: http://www.grupoisce.com.mx/servicios. php?content=seguros

9. Instituto de Energía. (2014). Requerimientos para el desarrollo del reservorio de Vaca Muerta (Neuquén/ Argentina). Buenos Aires: Academia Nacional de Ingeniería.

10. Mawet, P., Fleming, A. C., \& Nichols, J. H. (2012). Eight leading practices for the proppant suply chain. Accenture, 1-19.

11. Nodo Logístico. (08 de Diciembre de 2010). Tabla de fletes. Obtenido de Nodo Logístico: http://www. nodologistico.com/?p=492

12. PacWest Consulting Partners. (2014). North America Frac Sand Outlook. Eau Claire, WI: PacWest Consulting Partners.

13. Palisade Corporation. (2013). Guía para el uso de @ Risk: programa de complemento para el análisis y simulación de riesgos en Microsoft Excel. Nueva York: Palisade Corporation.

14. Proexport Colombia. (27 de Noviembre de 2013). Tarifas por Rutas- Transporte Marítimo. Obtenido de ProColombia: http://www.colombiatrade.com. co/herramientas/logistica/reportes-de-transporte/ maritimo/tarifas-por-rutas 
15. Revista Petroquimica. (10 de Octubre de 2014). Halliburton inaugura la primera planta de almacenamiento y distribución en la Argentina. Obtenido de Revista Petroquimica: http:// revistapetroquimica.com/halliburton-inaugura-laprimera-planta-de-almacenamiento-y-distribucionen-la-Argentina/

16. Sáchica, J., \& Amaya, R. (2010). Análisis técnicoeconómico de los fracturamientos hidráulicos realizados en los Campos Llanito, Gala y Galán de Ecopetrol S.A. Bucaramanga: Universidad Industrial de Santander .

17. Serrano, D., Pineda, C. A., Corzo, R., \& Castillo, R. D. (2013). Implementación de propantes ultralivianos en el fracturamiento hidráulico de pozos. Fuentes: El Reventón Energético, 5-16.

18. Sovacool, B. K. (2014). Cornucopia or curse? Reviewing the costs and benefits of shale gas hydraulic fracturing (fracking). Renewable and
Sustainable Energy Reviews, 249-264.

19. Superintendencia de Vigilancia y Seguridad Privada. (2014). Tarifa para la contratación de servicios de vigilancia y seguridad privada. Bogotá.

20. Toncel, E. A. (2013). Factibilidad técnica y financiera para la fabricación de material propante, base arena natural, utilizado en el fracturamiento hidráulico en Colombia. Bucaramanga: Universidad Industrial de Santander.

21. U308CORP. (2014). South American Silica Corp. aplica para obtener una concesión en Uruguay luego de haber recibido resultados postivos en las pruebas realizadas a las arenas a ser utilizadas dentro del proceso de fracturación hidráulica. Toronto: U308CORP.

22. Varela, R. (2010). Evaluación Económica de Proyectos de Inversión. Bogotá: McGraw Hill.

Recepción: 15 de febrero de 2016

Aceptación: 11 de abril de 2016 